\title{
An Accelerated Iterative Image Reconstruction Algorithm Based on Multiple Linear Regression for Direct 3D ECT
}

\author{
Yifan Wang, Hua Yan, Lijun Liu, Yinggang Zhou \\ School of Information Science and Engineering, Shenyang University of Technology, Shenyang, China \\ Email: yanhua_01@163.com,wyf2001315@163.com
}

\begin{abstract}
The image reconstruction for electrical capacitance tomography (ECT) is a nonlinear, underdetermined and illposed inverse problem. It is difficult to achieve a reconstructed image with higher quality, especially in 3D reconstruction. The study of image reconstruction algorithm is a crucial issue in ECT. In this paper, a novel algorithm of image reconstruction (MLRALI) applying to 3D ECT is presented. The basic idea behind this algorithm is to establish the forward problem by utilizing multiple-linear regression without utilizing sensitivity matrix, then achieve a stable solution of the inverse problem by utilizing an accelerated Landweber iteration algorithm. Extensive simulations have demonstrated that the algorithm proposed has better reconstruction performance compared with the Landweber iteration algorithm utilizing sensitivity matrix for direct 3D reconstruction.
\end{abstract}

Keywords- multiple linear regression; Landweber; 3D; ECT; image reconstruction

\section{INTRODUCTION}

Electrical capacitance tomography (ECT) is a relatively mature tomographic measurement technique in numerous different industrial tomography methods [1-2]. ECT technique obtains the measured capacitances of a multielectrodes sensor around an industrial container or pipeline including two materials of different dielectric constant. By these measurement values, the dielectric constant distribution of the $2 \mathrm{D}$ cross-section or in the $3 \mathrm{D}$ space can be reconstructed by applying an appropriate algorithm. Up to now in applications, ECT are used mainly in the monitoring of two-phase flows in industrial process, imaging flames in combustion, and gravitational flows in silo, etc [3-5]. It has been widely used for the advantages of non-invasive, fast response and low cost.

In the past few years, 3D ECT imaging [6-8] has gained increased attention. A 3D ECT sensor usually has at least two-plane measuring electrodes in an axial direction. The capacitance measurements are taken between any two measuring electrodes; that is to say, not only to measure capacitance in the same plane but also in different plane. 3D images are reconstructed directly from these measured capacitance data by using a suitable reconstruction algorithm [7-8]. In some papers, 3D ECT is also called electrical capacitance volume tomography (ECVT) [7-9] due to the fact that it can realize volumetric imaging.

In ECT system, the forward problem is to acquire the measurement capacitances which caused by a known distribution of the dielectric constant. Instead, the inverse problem is to determine the dielectric material (permittivity) distribution from the measurement capacitances. This process is called as image reconstruction.

The linear back-projection (LBP) and the Landweber iteration(LI) algorithms [10] are the representative and widely used algorithms in image reconstruction algorithms for ECT. LBP algorithm has the advantages of simple, rapid and easy to realize. But, the reconstructed images obtained by LBP are fuzzy, the effect of sharp smooth transition in different materials was showed. Thus, LBP can be utilized to image reconstruction on line, but the reconstructed images only give a qualitative analysis. As we all know, Landweber iterative algorithm is the most widely iterative methods used in ECT. This algorithm has the advantages that the numerical realization is correspondingly easy and simple, Moreover in most instances this method is very efficacious. However, from the perspective of the numerical optimization, it is only a change of the gradient descent method, and the convergent rate is relatively slower. The algorithm often begins to increase gradually after arriving at a local minimum, because of its semi-convergence characteristics. Another drawback of the LI algorithm is that it often produces a number of scattered artifacts, especially when it is used for direct 3D image reconstruction. These artifacts usually emerge at the sites with stronger sensitivity. Thus they are sometimes called "sensitivity-caused artifacts".

But these two reconstructed algorithms are all based on the sensitivity matrix to achieve image reconstruction. The ECT sensor has a "soft field characteristic", that is, the dielectric constant distribution of the measured material impact on the sensitive field distribution. The relationship between capacitance and dielectric constant is nonlinear and very complicated. If the analytical methods are used for ECT modeling, a lot of unreasonable and unrealistic assumptions must be used. For example, it assumes that measurement capacitances and permittivity distribution using the sensitivity distributions as the weighting factors are the linear relationship, and the assumption is clearly inconsistent with the actual. Multiple-linear regression analysis is a commonly tools utilized for statistical analysis. In this paper, multiple linear- regression analysis method is applied to study the functional relations between capacitance and permittivity distribution and establish statistical models.

In order to accelerate convergence speed of the reconstructed method and then improve the quality of reconstructed image, we propose a new algorithm in this 
paper. The main idea of the novel method is to establish the forward problem by applying multiple-linear regression (MLR) [11] method and then gain a stable solution of the inverse problem by utilizing the accelerated Landweber iterative algorithm. For convenience, this new algorithm is also called as MLRALI algorithm for short.

\section{LANDWEBER ITERATON ALGORITHM}

In ECT, the relationship between the measured interelectrode capacitance and the permittivity distribution of the region to be reconstructed is nonlinear, for the sake of simplicity, can be simplified as a linear modality $[10,12]$ :

$$
C=S G
$$

where, $C \in R^{N \times 1}$ is the normalized capacitance vector, $G \in R^{M \times 1}$ is the normalized dielectric constant vector, and $S \in R^{N \times M}$ is the Jacobian matrix of normalized capacitance in connection with the normalized dielectric constant, i.e. the normalized sensitivity matrix. The independent number of capacitance measurements $(N)$ is equal to $L(L-1) / 2$, where $L$ is the number of electrodes. The normalized permittivity is often called normalized grey level, and $G$ is often called grey vector too.

The Landweber iteration procedure is written as

$$
G_{k+1}=G_{k}+\alpha S^{T}\left(C-S G_{k}\right)
$$

where the step length $\alpha=2 / \lambda_{\max }$ is a constant value ( $\lambda_{\max }$ is the largest eigenvalue of $S^{T} S$ ), meeting the convergent condition of $\left\|\alpha S^{T} S\right\| \leq 2$.

\section{The New Reconstruction Algorithm-THE MLRALI ALGORITHM}

\section{A. Forward problem modeling by MLR}

An $N$-electrode ECT sensor can gives $N=L(L-1) / 2$ independent capacitance measurements. Supposing the imaging region in the pipeline is divided into $M$ small pixel element units by finite element (FE) method. we can obtain a group of $N$ capacitances when the sensor is filled with the lower dielectric constant material, a group of $N$ capacitances when the sensor is filled with the higher dielectric constant material, and $M$ groups of $N$ capacitances when the $p$ th unit inside the imaging region has higher permittivity and the rest units have lower permittivity, $p$ is $1,2, \ldots, M$ in proper order. The normalized values of capacitance were calculated by (3):

$$
\begin{array}{r}
\lambda(i, j)=\frac{C(i, j)-C_{l}(i, j)}{C_{h}(i, j)-C_{l}(i, j)} \\
i=1,2, \ldots, L-1, j=i+1, \ldots, N
\end{array}
$$

where $C_{l}(i, j)$ and $C_{h}(i, j)$ are the capacitances between electrode pair $i-j$ when the imaging region is respectively filled with lower or higher dielectric constant materials; $C(i, j)$ and $\lambda(i, j)$ are the measurement values of capacitance and the normalized values of capacitance for electrode pair $i-j$, respectively.

The capacitance value is normalized using (3) and then form a matrix $Q$ of $(M+2) \times N$ dimension. For $M$ pixel units, when the $p$ th element is filled by lower or higher permittivity material, the pixel $p$ is set to 0 or 1 . The $(M+2) \times M$ normalized matrix $F$ of dielectric constant distribution can be established. So multiple linear regression model between the normalized capacitance and the normalized distribution of media is followed:

$$
Q=F A_{0}+E
$$

where, $A_{0}$ is a $M \times N$ dimensional regression coefficient matrix; $E$ is the error matrix.

The least-squares estimate of $A_{0}$ is obtained by

$$
\hat{A}_{0}=\left(F^{T} F\right)^{-1} F^{T} Q
$$

The relationship between the capacitance values and the dielectric constant distribution can be gained and the forward model built by applying MLR can be written as

$$
\begin{gathered}
C=A G \\
A=\hat{A}_{0}
\end{gathered}
$$

where, $G$ is $M \times 1$ normalized matrix of pixel gray value; $C$ is $N \times 1$ matrix of normalized capacitance corresponding with the pixel gray $G$. $A$ is a $N \times M$ coefficient matrix, which the elements sum of each row is approximately equal to 1 . After the forward problem is established, the accelerated Landweber iterative algorithm is applied to solve the inverse problem.

\section{B. MLRALI algorithm}

Equation (6) is solved and the cost function to be minimized can be written as

$$
f\left(G_{k}\right)=\frac{1}{2} \omega_{k}\left\|C-A G_{k}\right\|^{2}
$$

In order to accelerate the convergent speed, we add an additional acceleration term into the cost function to be minimized. The new cost function formed can be written as

$$
F\left(G_{k}\right)=\frac{1}{2}\left\{\mu_{k}\left\|G_{k}-G_{k-1}\right\|^{2}+\omega_{k}\left\|C-A G_{k}\right\|^{2}\right\}
$$

The gradient of $F\left(G_{k}\right)$ with respect to $G_{k}$ can be simply calculated as

$$
\nabla F\left(G_{k}\right)=\mu_{k}\left(G_{k}-G_{k-1}\right)+\omega_{k} A^{T}\left(C-A G_{k}\right)
$$

So the iteration procedure becomes

$$
\begin{aligned}
G_{k+1} & =G_{k}+\nabla F\left(G_{k}\right) \\
& =G_{k}+\mu_{k}\left(G_{k}-G_{k-1}\right)+\omega_{k} A^{T}\left(C-A G_{k}\right) k \geq 0
\end{aligned}
$$

where, $G_{k}-G_{k-1}$ is the difference of permittivity distributions between the $k$ th step and $(k-1)$ th step, $C-A G_{k}$ is the remainder term between calculated and measured capacitances, the step sizes $\mu_{k}$ and $\omega_{k}$ can be viewed as weighted coefficient for them, respectively. We calculate $\mu_{k}$ and $\omega_{k}$ by using $v$-method [13]. 


$$
\begin{cases}\mu_{0}=0 & \\ \omega_{0}=\frac{4 v+2}{4 v+1} & k \geq 1 \\ \mu_{k}=\frac{k(2 k-1)(2 k+2 v+1)}{(k+2 v)(2 k+4 v+1)(2 k+2 v-1)}, & k \geq 1 \\ \omega_{k}=4 \frac{(2 k+2 v+1)(k+v)}{(k+2 v)(2 k+4 v+1)}, & k \geq 1\end{cases}
$$

The parameter $v(v>0)$ in the $v$-method is given by experience. In this paper, the value of $v$ is 1.5 .

For reducing iterative artifacts of the reconstructed image, we apply a threshold operation to the image reconstructed in each iteration. The formula of the MLRALI algorithm mentioned in the paper can be written as follows

$$
\left\{\begin{array}{l}
G_{-1}=0 \\
G_{0}=A^{T} C \\
G_{k+1}=P\left[G_{k}+\mu_{k}\left(G_{k}-G_{k-1}\right)+\omega_{k} A^{T}\left(C-A G_{k}\right)\right], k \geq 0 \\
P(x)= \begin{cases}0 & \text { if } x<\eta_{k} \\
x & \text { if } \eta_{k} \leq x \leq 1 \\
1 & \text { if } x>1\end{cases}
\end{array}\right.
$$

where, $\eta_{k}\left(0 \leq \eta_{k}<1\right)$ is the threshold value for the image reconstructed in $k$ th iteration. In this paper, the values of $\eta_{k}$ is 0.08 .

If $\eta_{k} \equiv 0$ in (13), that is, the adaptive threshold value is fixed at 0 in iteration and $A=S$, the MLRALI algorithm becomes accelerated Landweber iteration (abbreviated as ALI) algorithm. And if $A=S, \eta_{k} \equiv 0, u_{k} \equiv 0, \omega_{k} \equiv 2 / \lambda_{\text {max }}\left(\lambda_{\text {max }}\right.$ is the maximum eigenvalue of $S^{T} S$ ) in (13), the new algorithm becomes the well-known conventional Landweber iteration (abbreviated as LI) algorithm propose by Yang et al.

\section{DiRECT 3D ECT IMAGES RECONSTRUCTION}

Fig. 1 gives the structure diagrams of the 3D ECT sensor applied in simulation. This three-dimensional ECT sensor has twelve electrodes fixed symmetrically on the external surface of an insulated pipeline, give $66(L=12, N=66)$ independent capacitance measurements. These electrodes are placed in three planes, every plane has four electrodes and rotates $45^{\circ}$ according to the upper plane electrode. The Finite Element (FE) method was applied to calculate the capacitance simulation data. Fig. 2(a) shows the 3D FE mesh for this sensor. It has 12288 pentahedral elements and 4800 inside the pipe. The 3D FE mesh is acquired by the twodimensional mesh axial tension shown in Fig. 2(b) along axial direction. The whole 3D area is divided into 24 layers in axial direction. Layer 1 is in the bottom. Electrodes 1-4 occupy layers 3-6, electrodes 5-8 occupy layers 11-14, and electrodes 9-12 occupy layers 19-22.

Besides of the reconstructed images, relative image error $(R E)$ and correlation coefficient $(C C)$ are used to evaluate the reconstruction quality quantitatively.

$$
\begin{gathered}
R E=\frac{\|\hat{G}-G\|}{\|G\|} \\
C C=\frac{\sum_{p=1}^{M}\left(\hat{G}_{p}-\overline{\hat{G}}\right)\left(G_{p}-\bar{G}\right)}{\sqrt{\sum_{p=1}^{M}\left(\hat{G}_{p}-\overline{\hat{G}}\right)^{2} \sum_{p=1}^{M}\left(G_{p}-\bar{G}\right)^{2}}}
\end{gathered}
$$

where $G$ and $\hat{G}$ are the true and the reconstructed dielectric distribution of the test target respectively, $\bar{G}$ and $\overline{\hat{G}}$ are the average value of $G$ and $\hat{G}$, respectively, $p$ represents the $p$ th element unit in the imaging area, $M$ is the sum of number of the elements in the imaging area. Smaller

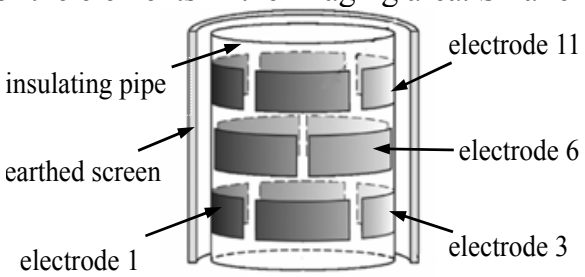

Figure 1. The 3D ECT sensor

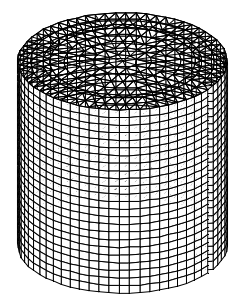

(a)

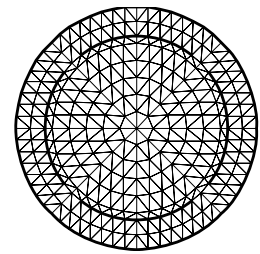

(b)
Figure 2. The FE mesh for the sensor: (a) the 3D FE mesh used in FE calculation; (b) the 2D FE mesh used for generating the 3D mesh shown in (a).

$R E$ and $C C$ closer to 1 mean better reconstructed quality.

Four representative permittivity distributions in $3 \mathrm{D}$ were used for testing the reconstruction performance, as shown in the first row of Table 1 below. The true distributions are one or two cylinder object which diameter and height are $18.4 \mathrm{~mm}$ and $9 \mathrm{~mm}$ respectively. The low (background) and the high (object) dielectric materials chosen were air (relative inductivity is 1) and perspex (relative inductivity is 2.6). Fig. 3 show the top and the main views of these four cases. The bold black lines on the right side of the main views represent the axial position of the electrode planes.

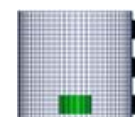

(I)

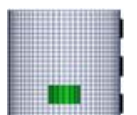

(II)

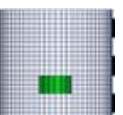

(III)

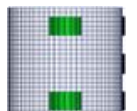

(IV)

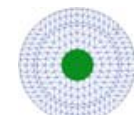

(V)
Figure 3. The main and the top views of four true distributions 

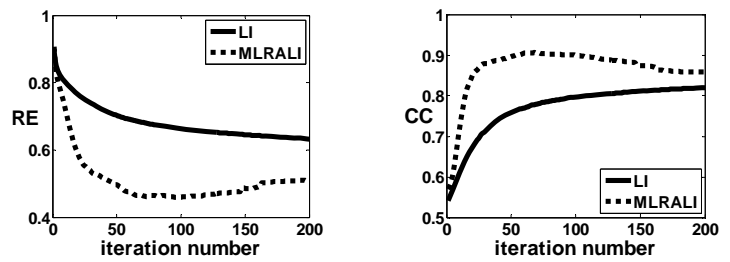

Figure 4. Evaluated error curves of true distributions I

The reconstructed images of LI with 200 iterations and MLRALI with 70 iterations are given in Table 1. As an example, the relative image error curve and correlation coefficient curve of the true distribution I obtained by the LI and MLRALI algorithms are given in Figure 4. Conclusions as follows can be obtained from Table 1 and Figure 4.

1) The reconstructed images of LI algorithm and MLRALI algorithm all can give the position and shape of the true objects.

2) The additional acceleration term can accelerate the convergence rate effectively. Compared with LI, MLRALI is faster in convergence speed.

3) The MLRALI is better than LI in reconstruction quality. Moreover, $R E$ and $C C$ of MLRALI algorithm are superior to the LI algorithm.

\section{CONCLUSION}

The direct 3D ECT imaging simulation is carried out in this paper. An 12-electrodes ECT sensor and 3D finite element model were used in this paper. The main idea which is given in this paper is to establish the forward problem by applying multiple-linear regression without using sensitivity matrix, then gain a stable solution of the inverse problem by utilizing an accelerated Landweber iterative algorithm. Reconstructed results show that the images reconstructed by LI and MLRALI can give the position and shape of true distribution. The MLRALI algorithm is better in reconstructed quality. Compared with the widely used LI algorithm, the MLRALI algorithm is better in reconstruction quality and faster in convergence speed.

\section{ACKNOWLEDGMENT}

The authors thank the National Natural Science Foundation of China for supporting this research under the contract No. 61071141 and the Program for Liaoning Excellent Talents in University under the contact No. LR2013005.

\section{REFERENCES}

[1] W. Q. Yang, "Design of electrical capacitance tomography sensors," Meas. Sci. Technol., vol. 21, Feb. 2010, pp.1-13, doi:10.1088/09570233/21/4/042001.

[2] M. Soleimani, C. N. Mitchell, and Banasiak, R, "Four-dimensional electrical capacitance tomography image using experimental data," Progress In Electromagnetics Research, vol. 90, 2009, pp.171-186, doi:10.2528/PIER09010202.

[3] J. C. Gamio, J.Castro, L.Rivera, J. Alamilla, F. Garcia-Nocetti, and L. Aguilar, "Visualisation of gas-oil two-phase flows in pressurized pipes using electrical capacitance tomography," Flow Measurement and Instrumentation, vol. 16, Apr. 2005, pp.129-134, doi:10.1016/j.flowmeasinst.2005.02.011.

[4] Y. T. Makkawi, P. C. Wright, "Electrical capacitance tomography for conventional fluidized bed measurements----remarks on the measuring technique," Powder Technology, vol. 148, Nov. 2004, pp. 142-157, doi: 10.1016/j.powtec.2004.09.006.

[5] H. G. Wang, P. R. Senior, R. Mann, and W. Q. Yang, "Online measurement and control of solids moisture in fluidised bed dryers," Chemical Engineering Science,vol. 64, Jun. 2009, pp.2893-2902, doi: 10.1016/j.ces.2009.03.014

[6] M. Soleimani, H. G. Wang, Y. Li, and W. Q.Yang, "A comparative study of 3D electrical capacitance tomography," Int. J. Information Systems Sci. vol. 3, 2007, pp. 292-306.

[7] R. Wajman, R. Banasiak, L. Mazurkiewicz, T. Dyakowski and D. Sankowski, "Spatial Imaging with 3D Capacitance Measurements," Meas. Sci. Technol. , vol. 17, Aug. 2006, pp.21132118,doi:10.1088/0957-0233/17/8/009

[8] F. Wang, Q. Marashdeh, L. S. Fan and W. Warsito, "Electrical Capacitance Volume Tomography: Design and Applications," Sensors, vol. 10, Mar. 2010, pp.1890-1917, doi:10.3390/s100301890.

[9] J. M. Weber, J. S. Mei, "Bubbling fluidized bed characterization using electrical capacitance volume tomography (ECVT)," Powder Technology, vol. 242, Jul. 2013, pp. 40-50, doi:10.1016/j.powtec.2013.01.044.

[10] W. Q. Yang, D. M.Spink, T. A.York, and H. McCann, “An imagereconstruction algorithm based on Landweber's iteration method for electrical-capacitance tomography," Meas. Sci. Technol., vol. 10, Oct. 1999, pp.1065-1069, doi:10.1088/0957-0233/10/11/315.

[11] H. Yan, L. J. Liu, H. Xu, F. Q. Shao, "Image reconstruction in electrical capacitance tomography using multiple linear regression and regularization," Meas. Sci. Technol., vol. 12, Dec. 2001, pp.575581, doi:10.1088/0957-0233/12/5/304.

[12] J. D. Jang, S. H. Lee, K. Y. Kim, and B. Y. Choi, "Modified iterative Landweber method in electrical capacitance tomography," Meas. Sci. Technol., vol. 17, Jun. 2006, pp.1909-1917, doi:10.1088/09570233/17/7/032.

[13] M. Hanke, "Accelerated Landweber iterations for the solution of illposed equations," Numer. Math., vol. 60, 1991, pp. 341-373. 
TABLE I. TRUE DISTRIBUTIONS, RECONSTRUCTED DISTRIBUTION S AND RECONSTRUCTION ERRORS

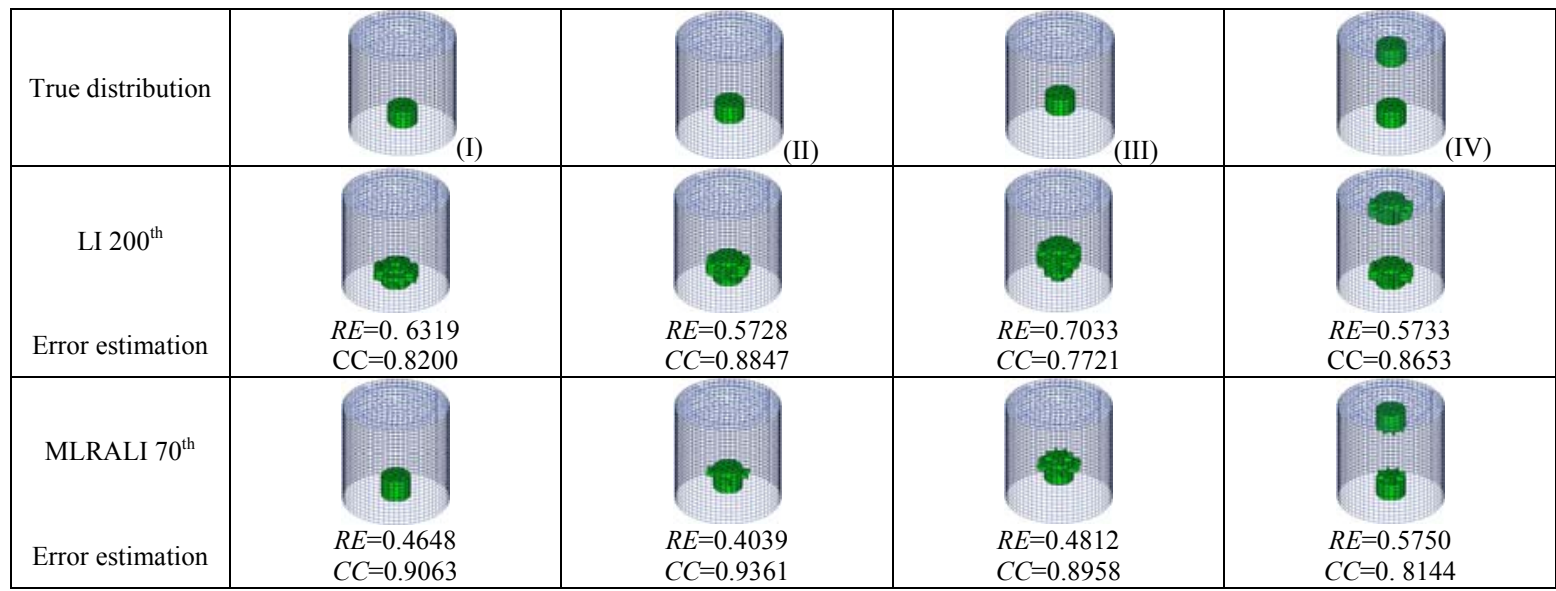

